\title{
Polymer Morphology as Dissipative Structure
}

\author{
P. H. LINDENMEYER \\ 165 Lee Street, Seattle, WA 98109, U.S.A.
}

(Received February 26, 1979)

\begin{abstract}
KEY WORDS Morphology / Dissipative Structure / Nonlinear Thermodynamics / Irreversible Processes /
\end{abstract}

The purpose of this note is to call to the attention of polymer physicists and morphologists the very exciting work on the thermodynamics of irreversible processes which has been coming out of the group of investigators at the University of Brussels. ${ }^{1-3}$ Since this group has been concentrating on the very large field of biological applications and since they have indicated that nonlinear irreversible thermodynamics is essentially a thermodynamics of chemical reactions, ${ }^{3}$ it is perhaps worth pointing out that phase transitions in polymeric materials also require nonlinear thermodynamics since the conformational entropy of a polymer molecule is a nonlinear function of the number of segments. Recognition of this fact makes it possible to use nonlinear irreversible thermodynamics to recognize polymer morphologies as dissipative structures rather than equilibrium structures and to begin their quantitative description. In this note we shall point out only some of the more obvious qualitative results of our initial exploration in the hope of persuading other polymer physicists to join in this exciting new approach.

All polymers crystallize (or solidify if crystallization is not possible) by irreversible processes. No matter how slowly crystallization takes place, the melting temperature of the resulting crystal is always substantially higher than the crystallization temperature. For example, Chiang and Flory ${ }^{4}$ carried out the crystallization of a polyethylene fraction at $131.3^{\circ} \mathrm{C}$ for over 40 days and found that the last trace of crystallinity did not disappear until $138.7^{\circ} \mathrm{C}$. The theoretical equilibrium melting point for polyethylene is generally accepted to be in the neighborhood of $145^{\circ} \mathrm{C}$. Clearly it is impossible to solidify polymeric materials reversibly in the time frame of normal laboratory operations. It follows that such solidification must be considered as an irreversible process occurring in a region far removed from equilibrium where the possibility of the formation of dissipative structures exists.

According to Glansdorff and Prigogine ${ }^{2}$ a dissipative structure will form when the steady state of an irreversible process becomes unstable with respect to a space-dependent fluctuation. These dissipative structures are significantly different from equilibrium structures and cannot be related by simple extrapolation. In contrast with equilibrium structures which are homogeneous and unlimited in size, dissipative structures are inhomogeneous and have characteristic size. Since they are fluctuations which have grown out of instabilities and are amplified and stabilized by the flow of energy and matter, the dissipative structures which evolve, unlike equilibrium structures, are dependent upon initial conditions as well as the complete history of events which occur during their existence. In effect, a nonhomogeneous structure with a characteristic size becomes a more efficient means for storing the excess energy of an irreversible process. What may appear to be a highly improbable fluctuation in molecular arrangement according to equilibrium thermodynamics becomes the arrangement which represents the combined thermodynamic and kinetic solution in the far from equilibrium case.

The process of solidification consists of the transformation of the kinetic energy of translational motion of the atoms or molecules in the liquid into some other form of energy. This energy is either (a) transformed into heat which flows to the environment, or (b) stored by increasing the internal energy of the solid. When the process is carried out reversibly (i.e., infinitely slowly), the heat which flows 
to the environment is the maximum amount and is called the latent heat of crystallization. When solidification is carrried out irreversibly, part of this heat is stored in the solid as excess energy in the form of randomly distributed defects and internal sufaces. Quantitatively, this excess energy is given by the temperature times the entropy production and is a function of both the kinetic fluxes and the thermodynamic forces of all the processes involved. A dissipative structure is formed whenever the translational kinetic energy of the liquid molecules can be more efficiently stored by the formation of a nonhomogeneous solid in which some of the energy liberated in one region is stored by the formation of highenergy structures in an adjacent region.

Thus the structure which forms when polymers solidify is really a dissipative structure which depends upon both the thermodynamics of the polymer molecule and the kinetics of the process by which solidification occurs. The structure is nonhomogeneous with a low-energy core, a high-energy surface and at least one characteristic dimension. If the polymer molecule is sufficiently regular and the entropy production is not too high, the low-energy core can evolve into a "true" crystal with threedimensional translational periodicity and the excess energy is concentrated in the noncrystalline regions on the surface. Thus we see that the basic error in the two-phase concept of polymer structure is not that two regions exist but that they represent separate structures. Actually they are both part of the same dissipative structure and are formed simultaneously since the one is needed to store the excess energy liberated by the formation of the other.

It is important to recognize that the so-called noncrystalline part of the dissipative structure is not just the "fronzen-in" random structure of the liquid, but represents a molecular arrangement which most efficiently stores the excess energy which must be retained in the solid as a consequence of the irreversible solidification process. Polymer molecules fold (in some manner) when they solidify since a fold is an efficient high-energy structure for storing the excess energy. The regularity of this fold will depend upon how much excess energy must be stored which in turn is quantitatively dependent upon the rate of entropy production. To argue that regular folding is unlikely on the basis of probability is like arguing that $10^{20}$ randomly moving water molecules will spontaneously begin to move in one direction while at some characteristic distance away an equal number will begin to move in the opposite direction. It is an event with almost zero probability according to classical statistics; yet it occurs invariably with the onset of convection cells whenever the rate of heating exceeds a critical value. Nonlinear irreversible thermodynamics is forcing a revolution on our ideas of statistical probability. ${ }^{5}$

Although Prigogine suggested the term " dissipative structures" a number of years ago, it was only recently recognized that a number of well-known phenomenon in the formation and behavior of polymer solids qualify for the use of the term "dissipative" to distinguish them from "equilibrium" structures. For example, the neck which is frequently observed during determation is a dissipative structure which forms when the force times the rate of deformation exceeds a critical value. The deforming system becomes unstable to a spacedependent fluctuation which evolves with time into a nonhomogeneous structure by minimizing the force at the expense of increasing the elongation (flux) in some regions and reducing it in others. The onset of this instability (i.e., the neck) is dependent upon both thermodynamics (i.e., the force) and kinetics (i.e., the rate of deformation).

Another example of a dissipative (nonequilibrium) structure is the dislocation. It minimizes the force necessary to deform a crystal by concentrating the flux along a narrow line. The dislocation illustrates another characteristic which we must expect in these nonequilibrium structures. When one no longer insists upon the homogeneity and the unlimited extent required by an equilibrium structure, the number of possible ordered arrangements is greatly increased beyond the 230 space groups of classical crystallography. Although the core of a dislocation is frequently referred to as "disordered" in comparison with the rest of the crystal, there is nothing random about the arrangement of the atoms within it. Each atom is arranged in a manner which most efficiently stores the excess energy which must be contained in the crystal as a result of the nonequilibrium process which created the dislocation.

In the same manner, there need be nothing random in the noncrystalline arrangements which form when polymers solidify by irreversible processes in the nonlinear region far from equilibrium. The molecular arrangement which form is that which 
most efficiently stores the excess energy and minimizes the thermodynamic forces.

The recognition of polymer morphologies as dissipative rather than equilibrium structures yields a very different insight upon what structures are to be expected and how to influence them. Instead of a homogeneous structure which minimizes the energy and maximizes the entropy, we must be concerned with a nonhomogeneous structure which most efficiently stores the excess energy while minimizing the thermodynamic forces. The excess energy which must be stored is given by the temperature times the entropy production which is the sum of the product of all the thermodynamic forces and their corresponding kinetic fluxes.

As the solidification process departs farther and farther from equilibrium the excess energy which must be stored increases, the characteristic distance decreases and the possibility of the formation of a true crystalline core decreases. When the entropy production is such that all of the translational kinetic energy of the liquid must be dissipated by the structure of the solid, solidification occurs by the formation of a glass. Thus a glass may be considered as the ultimate dissipative structure which may form upon solidification and the glass transition temperature may be quantitatively represented as the ratio of the latent heat of crystallization to the entropy production at the glass transition.

$$
T_{\mathrm{g}}=\Delta H_{\mathrm{c}} / \Delta_{i} S_{\mathrm{g}}
$$

Thus the glass transition, like entropy production, is a function of both thermodynamics and kinetics, a fact that is well-known experimentally. Recognition of a glass as a dissipative structure, however, permits one to consider it as two interpenetrating networks of high- and low-energy structures with a characteristic distance, and to calculate, in principle at least,

It is hoped that this brief note will serve to whet the appetitie of polymer physicists and inspire them to expend the effort necessary to understand nonlinear irreversible thermodynamics since it is clear that this is a science which controls polymer behavior in the solid state. ${ }^{7}$

the characteristic distance using the mathematics of spinodal decomposition. ${ }^{6}$

The question of "order" in so-called amorphous materials is beyond the scope of this brief note. However, it should be pointed out that dissipative structures are nonhomogeneous on the scale of their characteristic dimension and evolve from initial perturbations or fluctuations. Consequently, the "order" which might result from such a process will not be restricted to the traditional equilibrium crystal with its three-dimensional translational periodicity and its 230 space groups.

\section{REFERENCES}

1. I. Prigogine, "Introduction to Thermodynamics of Irreversible Processes," Intercience, New York, N.Y., 1967.

2. P. Glansdorff and I. Prigogine, "Thermodynamic Theory of Structure, Stability and Fluctuations," Wiley-Interscience, New York, N.Y., 1971.

The generality of the mathematical criterion for the onset of instability suggested by Glansdorff and Prigogine have been seriously questioned. (See for example B. H. Lavenda, "Thermodynamics of Irreversible Processes," Wiley, New York, N.Y., 1978), however, there is no question about the existence of these instabilities.

3. G. Nicolis and I. Prigogine, "Self-organization in Nonequilibrium Systems: From Dissipative Structures to Order through Fluctuations," WileyInterscience, New York, N.Y., 1977.

4. R. Chiang and P. J. Flory, J. Am. Chem. Soc., 83, 2857 (1961).

5. I. Prigogine, "Time, Structure, and Fluctuations," in Nobel Lectures 1977, Elsevier, Amsterdam, 1978, Science, 201, 777 (1978).

6. John W. Cahn, Acta Met., 9, 795 (1961); J. Chem. Phys., 42, 93 (1965), Trans. Met. Soc. AIME, 242, 166 (1968).

7. P. H. Lindenmeyer, "An Approach to the Theory of Polymer Solids" Wiley-Interscience, New York, N.Y., in preparation. 